\title{
Modelo Vectorial para la Inferencia del Estado Cognitivo de Pacientes en Estados Derivados del Coma
}

\author{
Esteban Velásquez R. ${ }^{1}$, Alejandro Cardona M. 1', Alejandro Peña P. ${ }^{1}$ \\ estebanvelaren@gmail.com, alejandro.cardona.m@hotmail.com,pfjapena@gmail.com \\ ${ }^{1}$ Grupo de Investigación en Ingeniería del Software y Modelamiento Computacional (GISMOC), Escuela de \\ Ingeniería de Antioquia, km 2+200 mts. Envigado, Colombia.
}

DOI: 10.4304/risti.13.65-81

\begin{abstract}
Resumen: La forma tradicional de evaluar el estado de conciencia de un individuo, ha sido mediante la aplicación de estímulos y el análisis de sus respuestas, sin embargo, esta técnica se ve limitada cuando el individuo es incapaz de responder evidentemente ante un estímulo, como es el caso de los pacientes en estados derivados del coma. En estos casos, se requiere de una conexión directa con el cerebro del paciente para detectar una respuesta. Por consiguiente, en este artículo se desarrolla y analiza un modelo computacional basado en los principios de las máquinas de soporte vectorial (MSV), para inferir el estado cognitivo de pacientes en estados derivados del coma, mediante la utilización de un equipo de electroencefalografía comercial. Los resultados obtenidos, mostraron que el modelo logró clasificar correctamente una tarea cognitiva en al menos cuatro de cada cinco pruebas en pacientes de control, lo que se traduce en la obtención de un sistema de bajo costo para el análisis del estado de conciencia y para la posible comunicación con algunos pacientes en estados derivados del coma mediante protocolos médicos definidos. De esta manera, este sistema se presenta como un gran aporte para las clínicas y centros hospitalarios, como herramienta potencial de diagnóstico para este tipo de pacientes.
\end{abstract}

Palabras-clave: Maquinas de soporte vectorial (MSV), Núcleo polinómico, Núcleo gaussiano, Electroencefalografía (EEG), Interfaz cerebro computador (ICC), Estado vegetativo.

\section{Vector Model for the Inference of the Cognitive State in Derived State Coma Patients}

Abstract: The traditional method to evaluate the conscious state of an individual consists in applying a stimulus and analyzing the response. However this technique is limited when the person cannot response evidentially to that stimulus, as are derived state coma patients. In such cases, a direct connection to the brain is required to detect the response. Therefore, in this paper we develop and analyze a computational model employing support vector machines (SVM) to infer the cognitive state of derived state coma patients using an affordable 
electroencephalography neuroheadset. The results given by the proposed model confirmed that the model can correctly classify the cognitive state in at least 4 out of 5 tests in control patients, which can be translated in the contribution of a low cost system for the analysis of the conscious state and the possible following communication with some derived state coma patients by defined medical protocols. Thus, this system is a contribution for clinics and hospitals as a potential diagnostic tool.

Keywords: Support vector machine (SVM), Polynomial kernel, Gaussian kernel, Electroencephalography (EEG), Brain-computer-interface (BCI), Vegetative state.

\section{Introducción}

Las estrategias de identificación de los niveles de conciencia en pacientes en estados derivados del coma, son actualmente escasas y limitadas. Tradicionalmente el estado cognitivo de un paciente, ha sido evaluado mediante la Escala de Coma de Glasgow, la cual mide la profundidad del coma en un estado postraumático agudo, basándose en la apertura ocular, la respuesta motora y la respuesta verbal (Carrión, Domínguez, \& Domínguez-Roldán, 2001). Sin embargo, esta escala no evalúa detalladamente el nivel de conciencia, sino que lo clasifica en un rango de gravedad que se fundamenta únicamente en respuestas motrices evidentes. Es por esto que una de las grandes limitantes de esta evaluación, está centrada en la incapacidad de realizar un diagnóstico más preciso y profundo acerca del estado cognitivo de un paciente postraumático (Cruse, et al., 2011).

La diferencia entre un paciente en estado vegetativo, y un paciente en estado de mínima conciencia, radica en la capacidad de evidenciar una respuesta consciente por parte del mismo. Es por esto que este segundo estado, se considera como una evolución del primero (inconsciencia), pero en muchos casos esta evolución no se logra diagnosticar correctamente, debido a que el paciente es incapaz de generar una respuesta motriz. Es por esto que actualmente, se está intentando integrar dispositivos especializados para medir la actividad cerebral de una persona, entre los que se cuentan las señales electroencefalográficas (EEG) (SSM Health Care, 2012), las imágenes por resonancia magnética funcional (IRMf) (Psych Central, 2008), la magnetoencefalografía (MEG) (Universidad Complutense de Madrid, 2001) y la tomografía por emisión de positrones (TEP) (MedlinePlus, 2013). Estas técnicas además están siendo utilizadas como soporte para el desarrollo de técnicas de evaluación de seguimiento de comandos cognitivos, es decir, tareas cerebrales que permiten analizar el grado de conciencia en los pacientes que poseen estas limitaciones físico-motrices (Schiff, 2002).

Algunos de los estudios más relevantes en esta área del conocimiento, hacen referencia al neurocientífico Adrian M. Owen de la Universidad de Cambridge, quien mediante imágenes de resonancia magnética funcional (IRMf) y estímulos auditivos, logró evidenciar una respuesta cognitiva en pacientes comatosos luego de que se les indicará que imaginaran dos actividades diferentes. Estas actividades conllevan un registro cerebral con patrones opuestos, los cuales pueden ser equiparados a un sí, o a un no, por parte del paciente. De esta forma, Owen logró identificar un nivel de conciencia en los pacientes evaluados (Owen, et al., 2006). Estudios posteriores realizados por este 
mismo neuroscientífico, validan los procedimientos realizados en este tipo de pacientes a partir de la demostración de niveles de atención específicos en algunos pacientes vegetativos y mínimamente conscientes (Owen, et al., 2013).

Otros avances en el área, especialmente relacionados con la electroencefalografía, hacen referencia al neuroscientífico Ryan D'Arcy, quien mediante una interfaz cerebrocomputador (ICC) de referencia ENOBIO, logró comunicarse con un paciente diagnosticado como vegetativo luego de sufrir un ataque cardíaco, a partir del registro de su actividad bioeléctrica cerebral (D’Arcy, 2012).

En consecuencia, en este artículo se desarrolla y analiza un modelo computacional basado en los conceptos de las máquinas de soporte vectorial (MSV), para la inferencia del estado cognitivo en pacientes comatosos, a partir de un conjunto de señales cerebrales EEG obtenidas mediante una interfaz cerebro computador (ICC) comercial, y basándose en los postulados de Owen (Adrian M Owen, 2004). Para tal efecto, en una primera etapa se seleccionó la ICC de acuerdo con una serie de criterios de accesibilidad, para luego proceder con la selección de las tareas cognitivas más adecuadas que produjesen patrones de activación cerebral diferenciados en los pacientes. Luego de conocer las dos tareas cognitivas, se continuó con la adquisición de las señales EGG a través de una serie de pruebas realizadas en pacientes de control (voluntarios sin ningún tipo de detrimento cognitivo); para tal fin se les indicó que imaginaran en un primer caso situaciones relajantes, y en un segundo caso situaciones frustrantes; finalmente se realizó el pre y post-procesamiento de los datos, así como la identificación de los patrones de activación mediante la utilización de la computación vectorial basada en las máquinas de soporte vectorial.

Los resultados obtenidos a través de la validación del modelo, demuestran un alto porcentaje de asertividad en la asociación de las tareas cognitivas propuestas, con relación a los registros electroencefalográficos de cada prueba, lo que se resume como un modelo tentativo para el trabajo con pacientes en estados derivados del coma en el estudio del grado de conciencia y la posterior comunicación con algunos de éstos, mediante protocolos médicos semejantes a los seguidos por los neurocientíficos mencionados anteriormente.

\section{Metodología}

La necesidad de conocer de una manera más profunda el estado cognitivo de pacientes en estado vegetativo, representa un gran reto para la medicina moderna, ya que esto ayudaría en gran medida al correcto diagnóstico y tratamiento de este tipo de pacientes. Es por esto que para mejorar este diagnóstico, se hace necesario el desarrollo de modelos que permitan el análisis de la respuesta cerebral de una persona ante una serie de comandos que demandan determinados patrones de activación.

\subsection{Selección de la ICC (Brain computer interface)}

Para la captura de la actividad bioeléctrica cerebral de un paciente en estado de coma, se procedió en primera instancia a llevar a cabo una amplia revisión en el mercado de diferentes tipos de ICC comerciales. Para tal efecto, se identificaron tres fabricantes líderes de este tipo de dispositivos a nivel mundial, entre los que se consideraron el 
Emotiv (Khushaba, et al., 2012), el Neurosky (Poltavski, Biberdorf, \& Petros, 2012) y el Neuroelectrics (Neuroelectrics, 2013). Posteriormente se procedió con la selección de uno de estos dispositivos, teniendo en cuenta para ello criterios técnicos tales como: número de canales, acceso a los registros puros de las señales EEG, así como el precio de mercado. Sin embargo, uno de los criterios de más peso para esta selección, estuvo determinado por el precio en el mercado, ya que una ICC comercial de bajo costo, mejoraría ostensiblemente el acceso de pacientes con bajos recursos a este tipo de diagnóstico.

\subsection{Registro de la actividad cerebral en pacientes de control}

Para obtener el registro de la actividad cerebral de un paciente en estado de coma, se procedió a establecer dos patrones cognitivos, en donde cada patrón estaba definido en términos de una tarea cognitiva, la cual consiste en un estímulo auditivo que orienta al paciente hacia un estado de relajación para un patrón, y uno de frustración para el segundo patrón.

Cada uno de estos patrones de activación fue aplicado a un conjunto de 30 pacientes de control. De esta forma, se le indicó a un paciente que se relajara por un período de tiempo corto, y en un segundo caso se le dijo que imaginara una situación frustrante. A partir de estos estímulos, se procedió a la recolección de la actividad cerebral del paciente, teniendo en cuenta para ello la ICC identificada en la etapa anterior, para lo cual se dispuso de un conjunto de 14 electrodos ubicados en el cuero cabelludo del paciente, en donde cada uno arroja como resultado una señal electroencefalográfica (EEG). Es de anotar que cada ICC en particular, utiliza una distribución específica de los electrodos en la cabeza de una persona, entre estas distribuciones se destacan la distribución Illinois, Montreal, Aird, Cohn, Lennox, Merlis, Oastaut, Schwab, Marshall, pero el más común es el sistema internacional "Diez-Veinte" (Hippe, Kulikowski, \& Mroczek, 2012).

Para lograr la diferenciación entre estas tareas en términos de los registros cerebrales obtenidos, se procedió con un análisis estadístico ANOVA, que para el caso particular de este trabajo, permitió determinar la diferencia o la semejanza entre los dos patrones establecidos. Estas dos tareas, permitieron además identificar por lo menos dos tipos de respuesta en un paciente de control, y con esto posibilitar una posterior comunicación básica con un paciente cualquiera a partir de un protocolo predefinido.

\subsection{Procesamiento de las señales EEG}

Con los registros EEG obtenidos de los pacientes de control, se procedió a realizar un preprocesamiento de los datos mediante la utilización de filtros pasa alto y pasa bajo, esto con el fin de atenuar los ruidos de baja frecuencia en las señales, como los que se presentan por debajo de $1 \mathrm{~Hz}$, y los de alta frecuencia, como los que se presentan por encima de los $50 \mathrm{~Hz}$. Así mismo se procedió con la eliminación de la línea base de las señales (offset), con el fin de eliminar tendencias ascendentes o descendentes. Por último se realizó un análisis de componentes independientes (ICA), con el objetivo de identificar y eliminar los artefactos biológicos presentes en los registros. Este preprocesamiento se llevó a cabo mediante la utilización de la herramienta de software EEGLab de MATLAB, el cual fue desarrollado por el Centro Swartz para la 
Neurociencia Computacional (SCCN, por sus siglas en inglés) (Neuroscience, 2014). Esta herramienta además de permitir la aplicación de los filtros mencionados anteriormente, permitió llevar a cabo diferentes tareas de visualización, edición y tratamiento de los registros EEG.

Luego del preprocesamiento, se procedió con la determinación de las potencias relativas de cada banda de frecuencia para cada una de las 14 señales EEG de activación cerebral de la siguiente forma: primero se realizó un periodograma modificado mediante una función de ventaneo de Hann, esto con el fin de evitar las discontinuidades al inicio y al final de la serie de datos obtenida en cada prueba (Jokinen, Ollila, \& Aumala, 2000). Con la nueva serie de datos obtenida (frecuencia vs potencia) se procedió a convertir las potencias en decibelios, logrando determinar la potencia en cada banda de frecuencia para las señales EEG (delta, teta, alfa, beta y gama).

En la figura 1, se muestra el espectro de potencia de las señales EEG obtenidas luego de realizar el procesamiento previo sobre los registros obtenidos en cada una de las pruebas. Es de anotar que para cada espectro de potencia, se determinaron las potencias relativas para cada banda de frecuencia e igualmente se realizó un promediio de las potencias para cada rango específico teniendo en cuenta una frecuencia de muestreo específica.

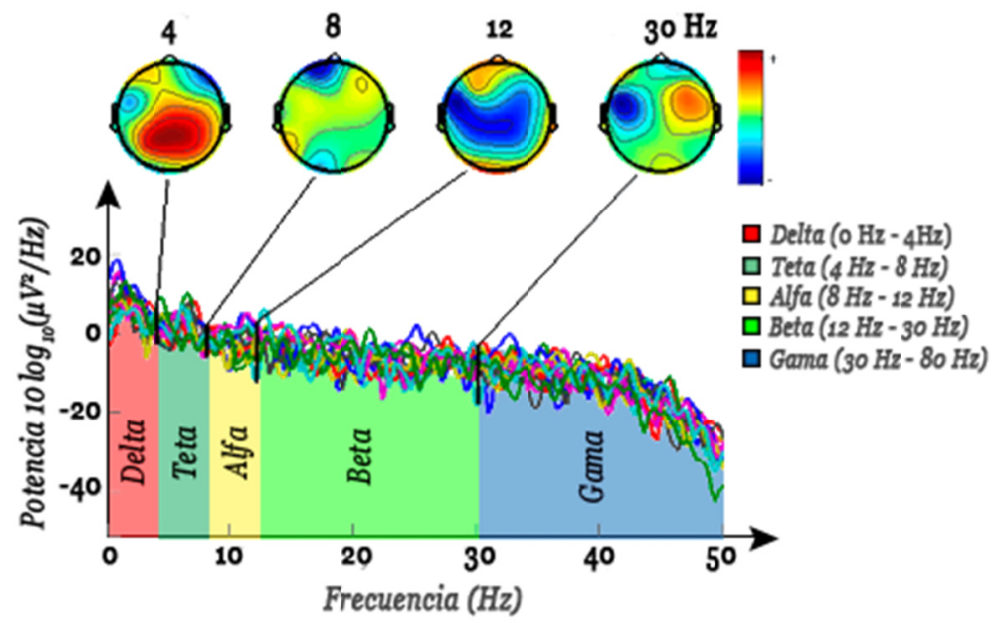

Figura 1 -Espectro de potencia de las señales EEG.

\subsection{Desarrollo del modelo de clasificación.}

De acuerdo con los patrones establecidos anteriormente, se desarrollaron dos máquinas de soporte vectorial (MSV) (Rojas, 1996), una primera definida por un núcleo polinómico, mientras que una segunda MSV estuvo determinada por un núcleo gaussiano. Cada una de estas MSV tomó como entradas las potencias en cada banda de frecuencia para el registro de una actividad cerebral, mientras que la salida estuvo determinada por las tareas cognitivas a identificar (frustración o relajación). Para el 
entrenamiento y la validación de los modelos propuestos, se llevó a cabo un estudio sobre 60 pacientes de control, el cual tiene en cuenta la siguiente estructura (figura 2).

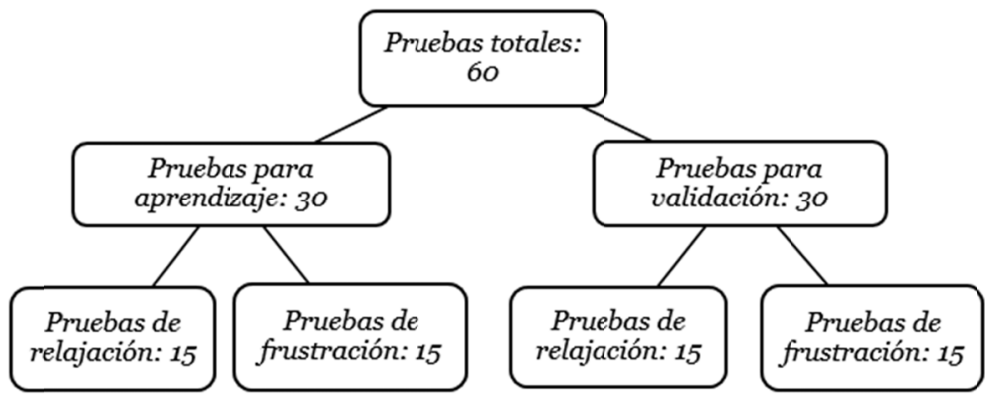

Figura 2 - Disposición de las pruebas para la identificación y validación de las MSV.

El entrenamiento de las MSV, se realizó mediante la utilización de la Regla Delta Generalizada (Isasi, 2004), esto con el fin de modificar las ponderaciones sinápticas (pesos) en función de la clasificación de los estados cognitivos utilizados como referencia. El modelo computacional de clasificación propuesto, fue desarrollado mediante la utilización de la programación orientada por objetos basada en tecnologías .NET, y su interoperabilidad de operaciones con MATLAB.

\section{Modelo vectorial con núcleo polinómico.}

La arquitectura de la MSV de tipo polinomio, estuvo determinada por un total de cinco neuronas en la capa de entrada, las cuales corresponden con cada una de las potencias promedio normalizadas (o a 1) en cada banda de frecuencia. Esta MSV posee una capa oculta definida por una serie de nodos, los cuales realizan una suma ponderada de lasi entradas, así como un nodo en la capa de salida definido por la función limitador duro, la cual determina los estados cognitivos definidos como referencia para cada registro de entrada.

De esta manera, una máquina de vector soporte de tipo polinómico se denota y define:

$$
\begin{gathered}
s_{k}(j)=\left(x_{k}(j) \cdot w_{k}(j, i)\right)^{d} \\
y r_{k}=f\left(c_{k}(j) \cdot s_{k}(j)\right)
\end{gathered}
$$

Donde:

$i$ : Indica el subíndice para las entradas.

j: Indica el subíndice para los nodos internos.

$k$ : Indica el número de registros utilizados para el aprendizaje. 
$\boldsymbol{x}_{\boldsymbol{k}}$ : Indica el vector $k$ de las potencias promedio en cada banda de frecuencia.

$w_{k}(j, i)$ : Indica las conexiones entre la capa de entrada y la capa oculta.

$S_{k}(j)$ : Indica los valores de salida de los nodos ocultos del modelo.

$c_{k}(j)$ : Indica las ponderaciones sinápticas entre los nodos del núcleo y la capa de salida.

$\boldsymbol{f}()$ : Indica la función limitador duro de la salida, el cual puede tomar el valor de 1 o o, dependiendo de la positividad del argumento de la función ( $\mathrm{O}=$ relajación, $1=$ frustración).

$\boldsymbol{y} \boldsymbol{r}_{\boldsymbol{k}}$ : Indica el valor de salida de la MSV.

$\boldsymbol{d}$ : Indica la potencia que define el núcleo polinómico.

La actualización de los pesos de la MSV, se hace en términos de la regla delta generalizada, para lo cual el error y la actualización de los pesos se denota y define (Isasi, 2004):

Donde:

$$
e_{k}=y d_{k}-y_{r k}
$$

$\mathrm{yd}_{\mathrm{k}}$ : Representa el estado de referencia de acuerdo con un estado cognitivo asociado a los registros de entrada.

$\mathrm{yr}_{\mathrm{k}}$ : Representa el valor de salida generado por la MSV para un estado cognitivo.

La actualización de las conexiones de la MSV mediante la aplicación de la regla delta generalizada:

$$
\begin{gathered}
\left.c_{k} \dot{(} J\right)=\propto \cdot e_{k} \cdot s_{k}(j) \\
\left.w_{k} \dot{(j}, \imath\right)=\propto \cdot e_{k} \cdot d \cdot c_{k}(j) \cdot\left(x_{k, i} \cdot w_{k, j, i}\right)^{d-1} \cdot x_{k, i}
\end{gathered}
$$

Dónde:

$\propto:$ Denota la velocidad en la actualización de las conexiones en la MSV.

\section{Modelo vectorial con núcleo gaussiano:}

La arquitectura de la MSV con núcleo gaussiano, está determinada igualmente como en el caso anterior, por cinco nodos de entrada que corresponden con cada una de las potencias promedio normalizadas (o a 1) en cada banda de frecuencia. El núcleo posee una capa oculta compuesta por una serie de nodos, los cuales estan definidos por una función de pertenencia gaussiana, e igualmente que en el caso anterior, esta MSV posee un nodo de salida definido por una función de activación limitador duro que corresponde con los dos estados cognitivos definidos para un paciente. De acuerdo con lo anterior, la MSV con núcleo gaussiano se denota y define: 
Donde:

$$
\begin{gathered}
S_{k}(j)=\frac{\left(\left(X_{k}(i)-X C_{k}(j, i)\right)^{2}\right)}{d^{2}} \\
H_{k}(j)=e^{-\frac{1}{2} \cdot S_{k}(j)} \\
y_{k}=c_{k}(j) \cdot H_{k}(j)
\end{gathered}
$$

$\mathrm{XC}_{\mathrm{k}}(\mathrm{j}, \mathrm{i})$ : Indica el valor de las componentes de los centroides que definen los nodos del núcleo gaussiano.

$\boldsymbol{d}_{\boldsymbol{k}}$ : Indica el tamaño de la base de cada uno de las funciones que determinan el núcleo gaussiano.

$\mathrm{Al}$ igual que en el caso anterior, la actualización de los pesos se realiza mediante la utilización de la regla delta generalizada, arrojando como resultado las siguientes ecuaciones:

$$
\begin{gathered}
\left.C_{k} \dot{(} \jmath\right)=\propto \cdot e_{k} \cdot H_{k}(j) \\
\left.W_{k} \dot{\jmath}, \imath\right)=2 \propto \cdot e_{k} \cdot C_{k}(j) \cdot H_{k}(j) \cdot X_{k}(j)
\end{gathered}
$$

\section{Análisis de resultados}

Para la selección de una ICC comercial identificadas en el mercado, se procedió a analizar una serie de características que son comunes entre diferentes ICC (Khushaba, et al., 2012), (Poltavski, Biberdorf, \& Petros, 2012), (Neuroelectrics, 2013), entre estas características se encuentran: número de canales, acceso a los registros de las señales EEG y Precio de Mercado. Para cada característica se estableció un puntaje de o a 10, en donde "o" representa la puntuación más baja, mientras que 10 representa la puntuación más alta. Los criterios para la asignación de dichos puntajes fueron los siguientes:

- Puntuación para Canales: o canales $->$ o puntos / 20 canales $->10$ puntos

- Puntuación para Acceso Registros Puros: No -> o puntos / Si -> 10 puntos

- Puntuación para Precio: USD $>=2000->0$ puntos,

$$
\text { USD }=0 \rightarrow 10 \text { puntos. }
$$

Tabla 1 - Especificaciones de las ICC por fabricante.

\begin{tabular}{|c|c|c|c|c|}
\hline \multicolumn{5}{|c|}{ Fabricante } \\
\hline \multicolumn{5}{|c|}{ Emotiv Systems } \\
\hline Referencia & Canales & Acceso registros puros & Precio & Puntuación \\
\hline$E P O C$ & 14 (7 puntos) & No (o puntos) & 299USD (8.5 puntos) & 15.5 \\
\hline$E E G$ & 14 (7 puntos) & Si (10 puntos) & 750USD (6.25 puntos) & 23.25 \\
\hline
\end{tabular}




\begin{tabular}{|c|c|c|c|c|}
\hline \multicolumn{5}{|c|}{ Fabricante } \\
\hline \multicolumn{5}{|c|}{ 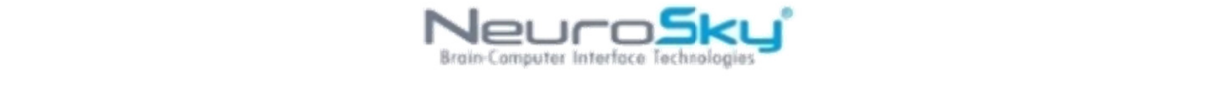 } \\
\hline Referencia & Canales & Acceso registros puros & Precio & Puntuación \\
\hline Mindwave & 1 (o.5 puntos) & No (o puntos) & 79.99USD (9.6 puntos) & 10.1 \\
\hline Mindset & 1 (o.5 puntos) & Si (10 puntos) & 199.99USD (9 puntos) & 19.5 \\
\hline Referencia & Canales & Acceso registros puros & Precio & Puntuación \\
\hline ENOBIO & 8 (4 puntos) & $\mathrm{Si}$ (10 puntos) & 5510USD (o puntos) & 14 \\
\hline ENOBIO & 20 (10 puntos) & Si (10 puntos) & 17233USD (o puntos) & 20 \\
\hline
\end{tabular}

De acuerdo con la tabla 1, y de acuerdo con la necesidad de obtener las señales EEG con la mejor resolución posible, se logró evidenciar que la ICC comercial más apropiada para el desarrollo de este sistema es la Emotiv EEG, la cual alcanzó un puntaje de 23.25 sobre 30 puntos posibles. Esta ICC, además de ser de fácil consecución en el mercado, posee un precio que es bastante aceptable con respecto a sus especificaciones técnicas. Es importante aclarar que las dos últimas ICC desarrollados por NE (Neuroelectrics $®$ ), poseen especificaciones técnicas superiores a las otras ICC de la misma casa, sin embargo su elevado precio de mercado es una limitante, ya que además de la funcionalidad, se pretende que este sistema sea de bajo costo.

Luego de la selección de la ICC, se procedió con la obtención de los registros cerebrales en los pacientes de control de acuerdo con la estructura propuesta en la figura 2. Estos registros se adquirieron a partir del protocolo de comunicación inalámbrico USB que el Emotiv EEG emplea, el cual es guiado por un software de la misma compañía que permite decidir cuándo y por cuánto tiempo se desea realizar la grabación EEG, para posteriormente visualizar y tratar los datos en el ordenador. De esta manera, en la figura 3 se puede observar la actividad bioeléctrica cerebral de un paciente, registrada en cada uno de los 14 electrodos definidos para el Emotiv EEG. Es de anotar que los electrodos frontales, centrales, temporales, parietales y occipitales, se nombran con las letras F, C, T, P y O respectivamente, mientras que el subíndice numérico, indica si el electrodo está situado en el hemisferio derecho (pares) o izquierdo (impares) de la cabeza del paciente. 


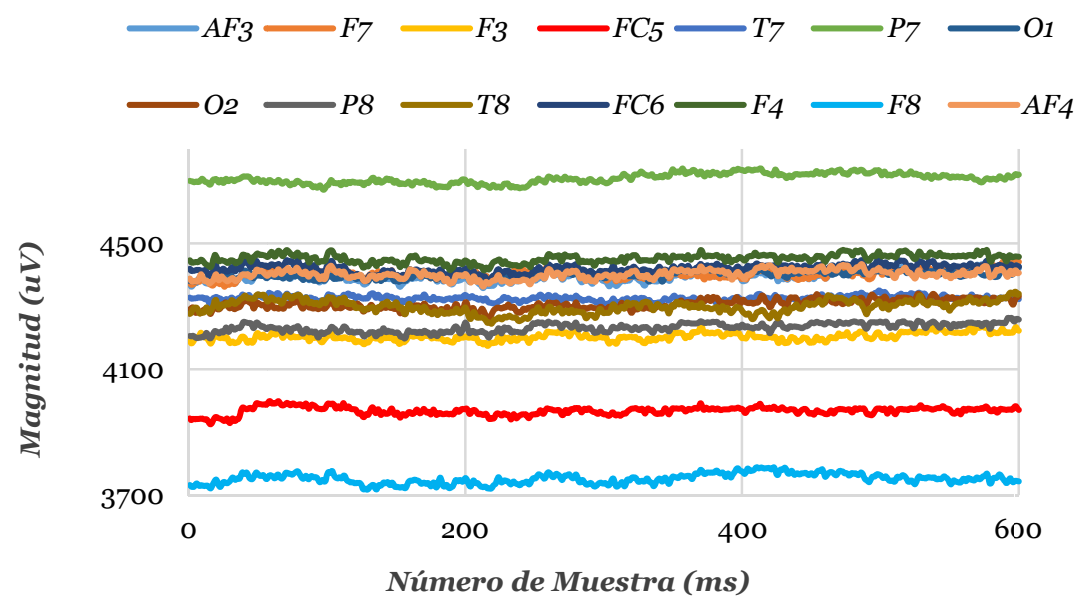

Figura 3-Electroencefalograma.

Para cada una de las señales, se obtuvo un total de 600 instantes de tiempo, que corresponden a un período de tiempo de 5 segundos. Conforme con la estructura de las señales, estas conformaron una matriz de $600 * 14$ datos. Las matrices en total de acuerdo con la estructura de pruebas fue de 60 , en donde 30 matrices correspondieron a relajación, mientras que las 30 matrices restantes, correspondieron al estado de frustración. En la figura 4, se pueden observar los patrones de frustración y relajación en términos de las señales EEG, en el dominio de la frecuencia, que registran la actividad cerebral de una persona.

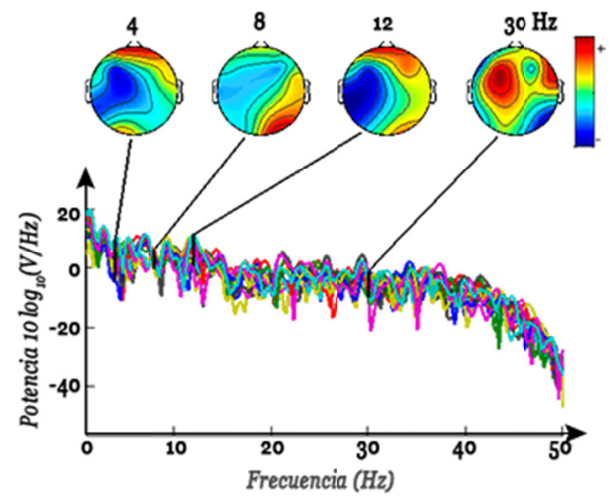

(a)

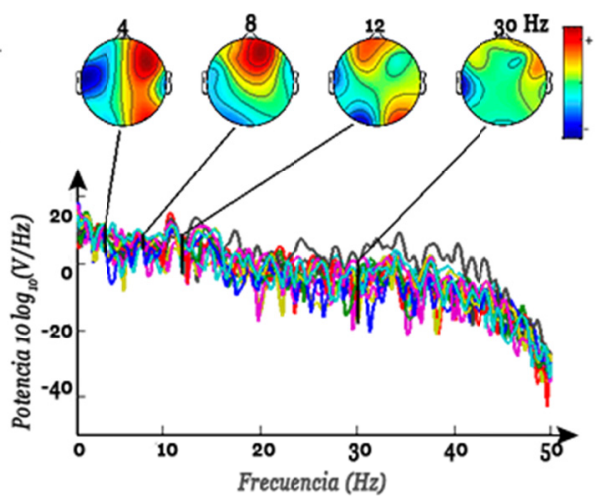

(b)

Figura 4 - Espectro de potencia en (a) patrón de relajación (b) patrón de frustración.

En la figura 4, se puede observar a priori, que la actividad bioeléctrica cerebral de una persona, es imposible de diferenciar a simple vista, por lo que se requiere de sistemas que permitan esta diferenciación. En las señales EEG obtenidas, se logró además evidenciar que algunas requieren de un tratamiento o preprocesamiento previo, con el 
fin de eliminar artefactos biológicos, ruido, offset y demás factores que puedan afectar la correcta interpretación de las señales. En la figura 5 (a) se puede observar un ejemplo de un artefacto biológico, el cual está definido por el movimiento de los ojos, haciendo que la potencia de las señales EEG se concentren en la parte frontal del cerebro. Este artefacto constituye uno de los principales factores de error a la hora de tratar las señales EEG, por lo que para su identificación y posterior eliminación, se recurre a un análisis de componentes independientes, que permite separar la señal de interés en diversos componentes y así determinar cuáles de estos son artefactos. En la figura 5 (b) se muestra la actividad del artefacto ocular en cuestión 1ms antes, hasta 2 ms después de ocurrido el evento (Neuroscience, 2014).

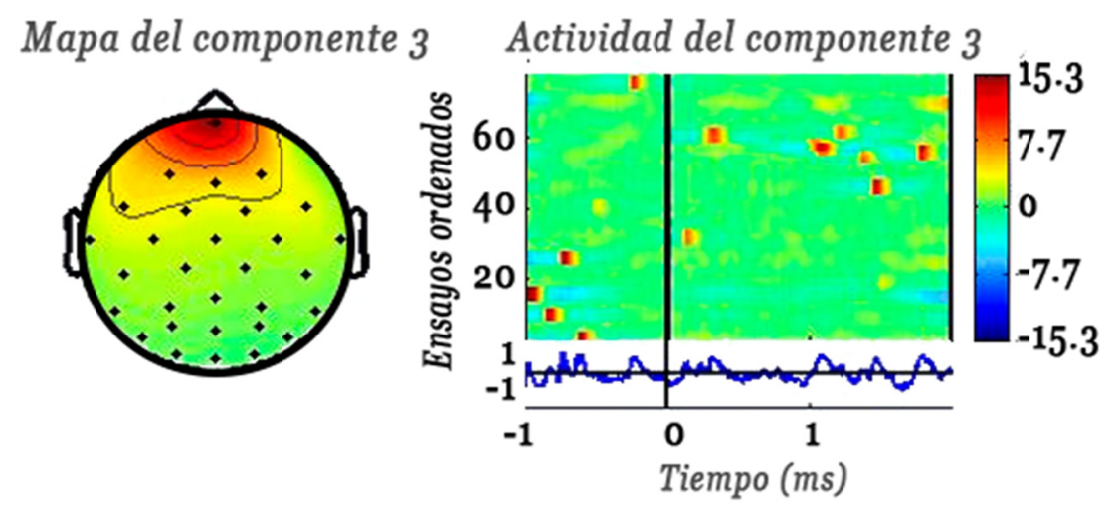

(a)

(b)

Figura 5 - Ejemplo de un artefacto biológico ocular (Neuroscience, 2014).

Luego de realizar el preprocesamiento y posterior procesamiento de los datos, se procedió a la obtención del espectro de potencia en cada una de las bandas de frecuencia (Delta, Teta, Alfa, Beta y Gama), arrojando como resultado una matriz de 6 (bandas) * 14 (electrodos) (ver tabla 2). Para cada matriz se procedió igualmente al cálculo de los promedios de los registros para cada banda, mostrando así que las potencias más altas se presentan en las bandas de frecuencia más bajas, mientras que en la banda Delta se presenta el valor de mayor potencia.

Tabla 2 - Potencias promedio de las señales EEG.

\begin{tabular}{|c|c|c|c|c|c|c|c|c|c|c|}
\hline \multicolumn{11}{|c|}{ Potencia (dB W) } \\
\hline Banda & $A F_{3}$ & $F_{7}$ & $F_{3}$ & $\mathrm{FC}_{5}$ & $\ldots$ & FC6 & $F_{4}$ & F8 & $A F_{4}$ & Promedio \\
\hline Delta & 17.69 & 11.30 & 14.77 & 10.15 & $\ldots$ & 13.04 & 14.38 & 14.05 & 17.22 & 14.64 \\
\hline Teta & 14.27 & 9.34 & 12.73 & 5.29 & $\ldots$ & 13.05 & 15.10 & 11.95 & 15.02 & 11.78 \\
\hline Alfa & 11.46 & 7.85 & 9.57 & 8.38 & $\ldots$. & 13.56 & 13.67 & 13.07 & 12.73 & 11.26 \\
\hline Beta & 11.47 & 10.51 & 12.07 & 12.03 & $\ldots$ & 12.63 & 13.43 & 11.81 & 12.34 & 12.00 \\
\hline Gama & 5.59 & 5.80 & 6.93 & 6.04 & $\ldots$ & 9.15 & 6.90 & 6.89 & 7.48 & 6.68 \\
\hline Total & 60.49 & 44.80 & 56.07 & 41.90 & $\ldots$ & 61.43 & 63.48 & 57.78 & 64.79 & \\
\hline
\end{tabular}


De acuerdo con lo anterior, y con el objetivo de conocer si las dos tareas cognitivas presentaban una diferencia estadísticamente significativa en las potencias promedio para cada banda de frecuencia, se realizó un análisis ANOVA como se muestra en la tabla 3:

Tabla 3 - Análisis estadístico ANOVA (STATGRAPHICS, 2013).

\begin{tabular}{|c|c|c|c|c|c|c|c|c|}
\hline \multirow{5}{*}{$\begin{array}{c}\text { Banda } \\
\text { delta }\end{array}$} & \multirow{2}{*}{ Fuente } & \multirow{2}{*}{$\begin{array}{c}\text { Suma de } \\
\text { cuadrados }\end{array}$} & \multirow[t]{2}{*}{$G l$} & \multirow{2}{*}{$\begin{array}{c}\text { Cuadrado } \\
\text { medio }\end{array}$} & \multirow{2}{*}{ Razón-F } & \multirow{2}{*}{ Valor-P } & \multicolumn{2}{|c|}{$\begin{array}{c}\text { Diferencia } \\
\text { entre estados }\end{array}$} \\
\hline & & & & & & & Si & No \\
\hline & Entre grupos & 1.0069 & 1 & 1.0069 & \multirow{3}{*}{0.32} & \multirow{3}{*}{0.5767} & & \multirow{3}{*}{$\mathrm{X}$} \\
\hline & Intra grupos & 185.267 & 58 & 3.19426 & & & & \\
\hline & Total (Corr.) & 186.274 & 59 & & & & & \\
\hline \multirow{5}{*}{$\begin{array}{c}\text { Banda } \\
\text { teta }\end{array}$} & \multirow[t]{2}{*}{ Fuente } & \multirow{2}{*}{$\begin{array}{l}\text { Suma de } \\
\text { cuadrados }\end{array}$} & \multirow[t]{2}{*}{$G l$} & \multirow{2}{*}{$\begin{array}{c}\text { Cuadrado } \\
\text { medio }\end{array}$} & \multirow{2}{*}{ Razón-F } & \multirow[t]{2}{*}{ Valor-P } & \multicolumn{2}{|c|}{$\begin{array}{c}\text { Diferencia } \\
\text { entre estados }\end{array}$} \\
\hline & & & & & & & $S i$ & No \\
\hline & Entre grupos & 0.364344 & 1 & 0.364344 & \multirow{3}{*}{0.17} & \multirow{3}{*}{0.6787} & & \multirow{3}{*}{$\mathrm{X}$} \\
\hline & Intra grupos & 121.952 & 58 & 2.10261 & & & & \\
\hline & Total (Corr.) & 122.316 & 59 & & & & & \\
\hline \multirow{5}{*}{$\begin{array}{l}\text { Banda } \\
\text { alfa }\end{array}$} & \multirow{2}{*}{ Fuente } & \multirow{2}{*}{$\begin{array}{l}\text { Suma de } \\
\text { cuadrados }\end{array}$} & \multirow{2}{*}{$G l$} & \multirow{2}{*}{$\begin{array}{c}\text { Cuadrado } \\
\text { medio }\end{array}$} & \multirow{2}{*}{ Razón-F } & \multirow{2}{*}{ Valor-P } & \multicolumn{2}{|c|}{$\begin{array}{c}\text { Diferencia } \\
\text { entre estados }\end{array}$} \\
\hline & & & & & & & Si & No \\
\hline & Entre grupos & 157.046 & 1 & 157.046 & \multirow{3}{*}{37.28} & \multirow{3}{*}{0.0000} & & \\
\hline & Intra grupos & 244.327 & 58 & 4.21253 & & & $\mathrm{X}$ & \\
\hline & Total (Corr.) & 401.373 & 59 & & & & & \\
\hline & Fuente & Suma de & $G l$ & Cuadrado & Razón-F & Valor-P & $\begin{array}{l}\text { Dif } \\
\text { entr }\end{array}$ & $\begin{array}{l}\text { ncia } \\
\text { tados }\end{array}$ \\
\hline & & cuadrados & & dio & & & $S i$ & No \\
\hline beta & Entre grupos & 41.7655 & 1 & 41.7655 & & & & \\
\hline & Intra grupos & 57.4943 & 58 & 0.991282 & 42.13 & 0.0000 & $\mathrm{X}$ & \\
\hline & Total (Corr.) & 99.2599 & 59 & & & & & \\
\hline & Fuente & Suma de & $G l$ & Cuadrado & Razón-F & Valor-P & $\begin{array}{l}\text { Dif } \\
\text { entr }\end{array}$ & $\begin{array}{l}\text { ncia } \\
\text { tados }\end{array}$ \\
\hline$B$ & & & & & & & Si & No \\
\hline gama & Entre grupos & 33.5581 & 1 & 33.5581 & & & & \\
\hline & Intra grupos & 69.6107 & 58 & 1.20018 & 27.96 & 0.0000 & $\mathrm{X}$ & \\
\hline & Total (Corr.) & 103.169 & 59 & & & & & \\
\hline
\end{tabular}

Este análisis mostró, que el valor $p$ fue menor de 0.05 para las bandas Alfa, Beta y Gama, por lo que se puede afirmar con un 95\% de confianza, que existe una diferencia estadísticamente significativa entre las tareas cognitivas en por lo menos tres de las 
cinco bandas de frecuencia, lo que permite que las MSV sean utilizadas para la identificación del estado de conciencia en los pacientes de control utilizados para este estudio (STATGRAPHICS, 2013).

De esta manera, el proceso de aprendizaje de las dos MSV se realizó mediante el ingreso de las potencias promedio en cada banda de frecuencia para los 30 pacientes de control. La salida asociada con cada grupo de señales, estuvo determinada por la tarea cognitiva asociada (relajación $=0$ y frustración $=1$ ). En la tabla 4 , se pueden observar los resultados obtenidos por los modelos vectoriales luego del proceso de aprendizaje y validación.

Tabla 4 - Resultados del proceso de aprendizaje y de validación para cada MSV.

\begin{tabular}{|c|c|c|c||c|c|c|c|c|}
\hline \multicolumn{4}{|c||}{ Aprendizaje MSV polinómica } & \multicolumn{4}{c|}{ Validación MSV polinómica } \\
\hline Estado mental & Aciertos & Desaciertos & Total & Estado mental & Aciertos & Desaciertos & Total \\
\hline Relajación & 13 & 2 & 15 & Relajación & 12 & 3 & 15 \\
\hline Frustración & 12 & 3 & 15 & Frustración & 11 & 4 & 15 \\
\hline Total & 25 & 5 & 30 & Total & 23 & 7 & 30 \\
\hline Aprendizaje MSV con núcleo gaussiano & Validación MSV con núcleo gaussiano \\
\hline Estado mental & Aciertos & Desaciertos & Total & Estado mental & Aciertos & Desaciertos & Total \\
\hline Relajación & 13 & 2 & 15 & Relajación & 12 & 3 & 15 \\
\hline Frustración & 14 & 1 & 15 & Frustración & 13 & 2 & 15 \\
\hline Total & 27 & 3 & 30 & Total & 25 & 5 & 30 \\
\hline
\end{tabular}

De acuerdo con lo anterior, las MSV utilizadas para la identificación de los estados cognitivos de un paciente de control, arrojaron en su proceso de entrenamiento porcentajes de reconocimiento cercanos al $83 \%$ en promedio, lo que refleja el buen aprendizaje de las MSV. Por su parte el modelo MSV con núcleo gaussiano, evidenció porcentajes de reconocimiento mucho más altos y cercanos al 90\%, lo que se evidenció en una disminución pronunciada del error por parte de este modelo como se muestra en la figura 6. Con respecto a la validación, los modelos alcanzaron en promedio un $76 \%$ de reconocimiento, siendo la MSV con núcleo gaussiano la que obtuvo un mejor resultado con un valor cercano al 83\%. Este porcentaje de reconocimiento, se logró gracias a la capacidad de generalización del modelo, como resultado de su comportamiento en la etapa de aprendizaje. Es de anotar que el porcentaje de reconocimiento logrado por un modelo, se obtiene a partir de la relación existente entre la cantidad de aciertos totales, frente al total de las pruebas utilizadas tanto en la etapa de aprendizaje como de validación. 


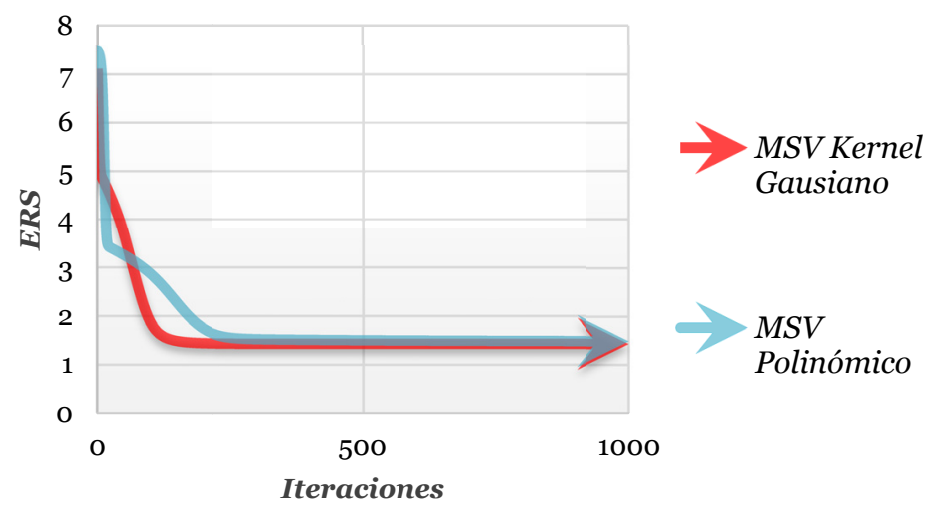

Figura 6 - Iteraciones vs Error para cada MSV en una prueba.

\section{Consideraciones y alcances}

Al momento de hacer las pruebas para la adquisición de los datos a tratar y analizar se pueden presentar diferentes factores de error que pueden comprometer la veracidad de los estudios y el alcance del modelo, tanto en los pacientes de control como en los pacientes en estados derivados del coma.

Para los primeros, estos factores suelen suceder debido a causas fisiológicas que no están relacionadas con un detrimento cognitivo o a causas externas que afectan la concentración; para los pacientes de control se deben tener en cuenta los factores enunciados a continuación:

- Los artefactos biológicos generados por movimientos oculares, tensiones musculares o palpitaciones, pueden alterar la señal a tratar.

- Se debe tener en cuenta que el estímulo auditivo inicial genera una respuesta cerebral que se evidencia en los registros EEG. Esto se conoce como P300, que es un potencial relacionado con eventos que ocurre $300 \mathrm{~ms}$ después del estímulo auditivo.

- En el momento de la prueba cabe la posibilidad de que el paciente no lleve a cabo adecuadamente la tarea cognitiva o piense en situaciones sin relación a esta.

- El paciente podría ser distraído de la tarea cognitiva asignada por alguna causa exterior.

Cuando se trata de pacientes en estados derivados del coma y se pretende realizar un electroencefalograma se deben considerar, además de los factores mencionados anteriormente, las siguientes consideraciones:

- La capacidad de estas personas para responder frente a un estímulo es fluctuante. 
- Es posible que en el instante de la prueba el paciente no se encuentre consciente, es decir, que esté en un estado de relajación profundo por un periodo de tiempo indeterminado.

- La dificultad que representa identificar los reflejos voluntarios de los involuntarios en la actividad cerebral del paciente.

- Presencia de déficits sensitivos, motores y cognitivos que imposibilitan una respuesta cerebral adecuada por parte del paciente frente a un estímulo.

- Se debe revisar si al paciente se le están suministrando medicamentos que puedan llegar a producir un estado de coma inducido farmacológicamente.

A partir de estas consideraciones, se debe tener presente que los resultados arrojados por el modelo no deben ser tomados como definitivos ya que hay una gran cantidad de factores subjetivos que se pueden presentar en el momento de las valoraciones, que influyen desde la posibilidad que existe de que el paciente olvide cómo realizar una determinada tarea cognitiva, que no sea capaz de llevarla a cabo, o que simplemente la prueba realizada para una tarea tenga el efecto contrario, como podría presentarse en el caso del estado de relajación, en donde existe el riesgo de que el paciente genere patrones de activación opuestos de forma involuntaria.

\section{Conclusiones}

Los modelos vectoriales propuestos, mostraron gran efectividad en la identificación de dos tareas cognitivas en pacientes de control. Sin embargo, el modelo MSV gaussiano se comportó de una mejor manera frente a la clasificación, ya que este modelo realiza una partición finita del espacio de solución del problema, a diferencia del modelo polinómico, el cual realiza la clasificación sobre un espacio infinito.

Existen diferentes factores de error que se pueden presentar durante las pruebas que influyen en forma determinante en la calidad de los registros ingresados al modelo, tanto para los pacientes de control como para los pacientes en estados derivados del coma. Esto supone la necesidad de tomar medidas de precaución de modo que se asegure la adquisición correcta de los registros electroencefalográficos y no se sesguen los resultados arrojados por el modelo.

La integración del modelo vectorial con las interfaces cerebro-computador de adquisición de señales EEG, permite generar un sistema de bajo costo que puede ser extendido de forma general a pacientes en estados derivados del coma a través del continuo perfeccionamiento del modelo con estudios posteriores en estos pacientes. De esta forma se posibilita la inferencia del estado cognitivo y la posterior comunicación con éstos a través de protocolos médicos definidos; sirviendo como herramienta de diagnóstico para las clínicas y centros hospitalarios.

\section{Referencias bibliográficas}

Adrian M Owen, S. L. (2004). Brain Function in Coma, vegetative State, and Related Disorders. The Lancet Neurology, Vol 3, 537-547. 
Carrión, J. L., Domínguez, M. d., \& Domínguez-Roldán, J. M. (2001). Coma y Estado Vegetativo: Aspectos médico-legales. Revista Española de Neuropsicología, 63-76.

Cruse, D., PhD, S. C., MSc, C. C., PhD, T. A., PhD, D. F.-E., MD, P. J., . . PhD, P. A. (2011). Bedside detection of awareness in the vegetative state: a cohort study. The Lancet, 378(9809), 2088-2094. Obtenido de http://www.thelancet.com/journals/lancet/article/PIISo1406736\%2811\%2961224-5/abstract

D’Arcy. (14 de Octubre de 2012). Consciousness scanner giving hope to brain trauma patients. CTVnews.

Emotiv. (7 de abril de 2013). Recuperado el 7 de abril de 2013, de http://www.emotiv.com/

Hippe, Z. S., Kulikowski, J. L., \& Mroczek, T. (2012). Human - Computer Systems Interaction: Backgrounds and Applications Vol.2. Rzeszów, Polonia: Springer.

Isasi, P. (2004). Redes de Neuronas Artificiales - Un enfoque práctico.

Jokinen, H., Ollila, J., \& Aumala, O. (2000). On windowing effects in estimating averaged periodograms of noisy signals. ScienceDirect, 197-207.

Khushaba, R., Greenacre, L., Kodagoda, S., Louviere, J., Burke, S., \& Dissanayake, G. (2012). Choice modeling and the brain: A study on the Electroencephalogram (EEG) of preferences. ScienceDirect, 12378-12388.

MathWorks.

(2002).

Obtenido

de http://www.ece.uvic.ca/ frodo/courses/elec26o/matlab/signal_tb.pdf

MedlinePlus. (2013).

Obtenido

de http://www.nlm.nih.gov/medlineplus/ency/article/o07341.htm

Neuroelectrics. (2013). Neuroelectrics. Obtenido de http://neuroelectrics.com/

Neuroscience, S. C. (2014). EEGLAB. Obtenido de EEGLAB: http://sccn.ucsd.edu/eeglab/

Neurosky. (7 de abril de 2013). Recuperado el 7 de abril de 2013, de http://www.neurosky.com/

Owen, A. M., Coleman, M. R., Boly, M., Davis, M. H., Laureys, S., \& Pickard, J. D. (2006). Detecting Awareness in the Vegetative State. Sciencemag, 1402.

Owen, A., Chennu, S., Finoia, P., Kamau, E., Monti, M., Allanson, J., . . . Bekinschtein, T. (2013). Dissociable endogenous and exogenous attention in disorders of consciousness. NeuroImage: Clinical, 450-461.

Poltavski, D., Biberdorf, D., \& Petros, T. (2012). Accommodative response and cortical activity during sustained attention. ScienceDirect, Pages 1-8.

Psych Central. (2008). Obtenido de http://psychcentral.com/lib/what-is-functionalmagnetic-resonance-imaging-fmri/0001056

Rojas, R. (1996). Neuronal Networks: A systematic introduction. 
Schiff, N. (2002). Residual cerebral activity and behavioural fragments can remain in the persistently vegetative brain. Brain, 1210-1234.

SSM Health Care. (2012). Obtenido de http://ssmhealth.adam.com/content.aspx?pid=5\&gid=003931

STATGRAPHICS. (2013).

Universidad Complutense de Madrid. (2001). Obtenido de http://pendientedemigracion.ucm.es/info/otri/complutecno/fichas/tec_tortiz1.ht $\mathrm{m}$ 